\title{
Crónica de una
}

\section{muerte silenciada.}

Racismo e

invisibilización en

\section{el asesinato de Massar}

\section{Ba* / Chronicle of a}

silenced death. Racism

and invisibility in the

murder of Massar Ba

* Artículo de reflexión. Recibido: 16 de septiembre de 2017. Aceptado: 10 de enero de 2018. Tla-Melaua, revista de Ciencias Sociales. Facultad de Derecho y Ciencias Sociales. Benemérita Universidad Autónoma de Puebla, México / issn: 1870-6916 / Nueva Época, año 12, núm. 44, abril-septiembre 2018, pp. 242-259.

Artículo de reflexión. Una versión anterior y reducida de este artículo ha sido presentada como ponencia en el III Congreso de Estudios Poscoloniales y IV Jornadas de Feminismo Poscolonial "Interrupciones desde el Sur: habitando cuerpos, territorios y saberes", unsaM, Buenos Aires. Ciertas preguntas y problemas planteados en este artículo surgen del "Conversatorio abierto Senegaleses en la Argentina: fronteras, luchas y derechos" del cual participaron Ndathie Moustafa Sene, presidente de la asociación de residentes senegaleses en la Argentina, el doctor Juan Ignacio Castien y la maestranda Lina Sanchez Alvarado. El evento fue organizado por el Grupo de Estudios Subalternidades en Latinoamérica (GESLAT) (del cual quien escribe forma parte) en septiembre del 2017 en homenaje a Massar Ba. A los organizadores, asistentes y panelistas, un profundo agradecimiento. 
El objetivo de este artículo es indagar cómo funciona el racismo en la ciudad de Buenos Aires, Argentina, la cual permite la producción política de la nuda vida, y la consolidación de un régimen de necropolítica en el que algunas vidas parecieran ser desechables. Se analiza el rol del Estado, los medios de comunicación y la sociedad en el asesinato de Massar Ba, quien fue golpeado hasta la muerte y abandonado en plena calle entre el 6 y el 7 de marzo del 2016. Entendemos este homicidio como un asesinato político y tipológico que nos enfrenta a lo más oscuro de nuestra sociedad y de la lengua que le sirve de soporte.

\section{PALABRAS CLAVE} Racismo, colonialidad del poder, Senegalese immigration, necro inmigración senegalesa, necropolítica. politics.
The objective of this article is to show how racism works in the city of Buenos Aires, Argentina, which allows the political production of nuda vida, and the consolidation of a necro political regime in which lives seem to be disposable. The role of the State, the media and society is analyzed in the murder of Massar Ba, who was beaten to death and left in the middle of the street sometime between March 6 and March 7, 2016. This homicide can be classified as a political assassination and typological that confronts us with the darkest side of our society and the language that supports it.

KEYWORDS

Racism, colonialism of power,

\footnotetext{
* Socióloga por la Universidad de Buenos Aires, maestranda en Estudios Sociales Latinoamericanos (MESLA), becaria doctoral del CONICET con sede en el Instituto de Estudios de América Latina y el Caribe (IEALC), miembro del Grupo de Estudios de Sociología Histórica de América Latina (GESHAL) y del Grupo de Estudios Subalternidades en Latinoamérica (GESLAT). (danarosenzvit@gmail.com) orcid.org/0000-0001-6988-3217
} 

del crimen: Buenos Aires, capital europea en América Latina / 4. El caso que nos compete / 5. Los sospechosos de siempre / 6. El veredicto / 7. Referencias

\section{EL CRIMEN}

En el ya clásico relato de Gabriel García Márquez, "Crónica de una muerte anunciada", Santiago Nasar es asesinado por una falta que no cometió. A plena luz del día y sin necesidad de esconder ni el arma ni el móvil, los hermanos Vicario llevan a cabo un homicidio que de antemano ya estaba en boca de todos. El pueblo entero se convierte en cómplice al no advertir a Santiago de lo que todos pensaban que seguro sabía. Lo que al principio es murmullo chismoso se convierte en alarido de espanto cuando presentes y ausentes caen en la cuenta del crimen colectivo.

Entre el 6 y el 7 de marzo del 2016, a Massar Ba, referente senegalés en Argentina, lo asesinaron a golpes en plena capital federal. Hacía meses que el conjunto de organizaciones representantes de los inmigrantes senegaleses en Argentina venía denunciando públicamente situaciones de maltrato policial, discriminación violenta, agresiones físicas y simbólicas y amenazas. Pero la noche en que mataron a Massar dio cuenta de que, aunque muchos sabían, nadie pensaba hacer nada. Frente al crimen consumado no iba a haber pueblo que gritara ni llorara sobre la sangre derramada. Lo que estaba a la vista de todos no estaría en boca de nadie, ni falta, ni arma, ni móvil, ni murmullo, ni alarido por un crimen que, por ciega, sorda y muda, mancha las manos de la sociedad toda.

Este artículo tiene como objetivo indagar en la manera en la que funciona el racismo en la Ciudad Autónoma de Buenos Aires, Argentina. Éste permite la producción política de la nuda vida, y la consolidación de un régimen de necropolítica en el que algunas vidas parecieran ser desechables. Sostenemos que el racismo en nuestra América Latina, y particularmente en Buenos Aires, debe ser leído y analizado teniendo en cuenta los tres tiempos braudelianos: la larga duración, al interior de la cual entendemos al racismo como un elemento estructural que data de la colonización y se ha convertido en una "realidad que el tiempo tarda enormemente en desgastar y que es sin embargo dinámico determinando el transcurrir de la historia"; la mediana

\footnotetext{
${ }^{1}$ Braudel, Fernand, "La larga duración", en Fernand Braudel, La historia y las ciencias sociales, Madrid, Alianza, 1970, pp. 70, 87.
} 
duración que implica el tiempo de la coyuntura; y por último, el tiempo del acontecimiento, en este caso el del asesinato de Massar Ba.

Para esto, combinaremos teorías posestructuralistas y poscoloniales para dar cuenta de la manera en que el asesinato que nos mancha a todos no fue una "muerte dudosa", como lo califica la causa penal que sigue estancada y en secreto sumario, sino el silencio en que los medios de comunicación hegemónicos, el Gobierno y las agrupaciones políticas incurrieron en torno al hecho. Evidencia que lo que pasó esa noche forma parte de un profundo entramado social que hace siglos oculta a los mismos a fuerza de violencia y desmemoria, de un sistema excluyente que a través del lenguaje y las prácticas cotidianas incurre en el crimen serial de la discriminación y la subalternización. Este crimen no se proscribe, porque se repite a diario, a fuerza de una costumbre que ya lleva, en palabras del cantautor argentino León Gieco, "cinco siglos igual".

\section{Antecedentes PEnAles}

En América Latina, el racismo ha sido, desde la colonización, un elemento estructural de larga duración, fundacional para la invención de América Latina en lenguaje europeo. La invención de la idea de la raza para nombrar y marcar desde Europa a sus otros colonizados y esclavizados fue necesaria para desarrollar los sistemas esclavistas americanos, los cuales suplieron la necesidad de mano de obra en las colonias tras el genocidio a las comunidades originarias. Los sistemas esclavistas por los cuales se importaron violentamente al menos quince millones de africanos cautivos desde las costas de África hasta América fueron "radicalmente novedosos en comparación con las formas previas de esclavitud". Su carácter comercial los convirtió en el "modelo" de los intercambios internacionales dentro del sistema-mundo desde el siglo XVI hasta el XIX, y las empresas que se organizaron para llevar a cabo la producción que en éstos se basaron. ${ }^{2}$

El sustento ideológico y cultural de este proceso fue el nacimiento del racismo propiamente moderno, por el cual por primera vez se identificó masiva y directamente a la esclavitud y a la raza. El color de piel funcionó como marca de pertenencia racial y como significante denigratorio; y generó la segregación necesaria para legitimar científicamente a la esclavitud. El positivismo y el evolucionismo funcionaron como pilares de esta división internacional del trabajo, y de la consiguiente construcción de pertenencias e ideales nacionales, característica privativa de y para los europeos. Al interior de la lógica de la acumulación del capital, el mito racial funcionó como ideología justificadora del genocidio y la esclavitud.

${ }^{2}$ Grüner, Eduardo, La oscuridady las luces. Capitalismo, cultura y revolución, Buenos Aires, Edhasa, 2010, p. 220. 
Las colonias americanas fueron organizadas a través de una jerarquía étnico-social en la que los blancos europeos ocuparon el vértice y los negros esclavos la base. A pesar del dinámico y necesario proceso de mestizaje que se desarrollaba entre ambos extremos de la estructura, las sociedades coloniales contaron con fuertes barreras raciales. Siguiendo a Fanon, podemos decir que "en las colonias, la infraestructura es igualmente una superestructura. La causa es consecuencia: se es rico porque se es blanco, se es blanco porque se es rico". 3 Siguiendo la misma lógica podemos parafrasear a Fanon: en las colonias "se es esclavo porque se es negro y se es negro porque se es esclavo". Las sociedades coloniales estuvieron definidas por la traducción de las diferencias raciales en diferencias sociales y por su soporte político y jurídico.

Foucault ${ }^{4}$ introduce la noción de biopolítica para pensar la política moderna en la que el racismo se convierte en el motivo indispensable para dar muerte a los otros. Esta es la condición gracias a la cual puede ejercerse el poder de matar. "El racismo entonces, instituye la diferencia sustantiva entre lo que debe vivir y lo que debe morir, pero, administrada por el Estado". ${ }^{5}$ Es en el espacio de la biopolítica que Agamben incluye a la nuda vida, presentada como "la figura en la que la vida humana se incluye en el orden jurídico únicamente bajo la forma de su exclusión, es decir de la posibilidad absoluta de que cualquiera le mate". ${ }^{6}$

Mbembe analiza la manera en que el "hacer vivir" en que se basa la biopolítica se transforma en un poder mortífero a través del cual impera la necropolítica, la cual subyuga a la vida frente al poder de la muerte. ${ }^{7}$ En cuanto se instaura el régimen necropolítico, el poder se despliega para establecer zonas permanentes de existencia en las sombras, grupos humanos disponibles para la muerte. De Oto y Quintana proponen utilizar estos conceptos para pensar el espacio colonial, el cual, insisten, es por excelencia el estado de excepción ${ }^{8}$ en que las poblaciones racializadas son reducidas a la nuda vida, donde están permanentemente disponibles para ser desechadas. El crimen, de larga duración en nuestro continente, tiene antecedentes penales en la explotación y el etnocidio de las poblaciones racializadas para ser esclavizadas.

Con las independencias latinoamericanas comenzó el proceso de formación de las naciones, a partir del mito europeo de la comunidad imaginada ${ }^{9}$ y a cargo de los Estados dirigidos por las elites blancas y criollas. La construcción

\footnotetext{
${ }^{3}$ Fanon, Frantz, Los condenados de la tierra, Buenos Aires, FCE, 1983.

${ }^{4}$ Foucault, Michel, Defender la sociedad, Buenos Aires, FCE, 2000.

${ }^{5}$ De Oto, Alejandro, Quintana, Maria Marta, "Biopolítica y colonialidad. Una lectura crítica de homo sacer", Tabula Rasa, enero-junio, 2010, p. 53.

${ }^{6}$ Agamben, Giorgio, Homo sacer. El poder soberano y la nuda vida, Valencia, Pre-textos, 2003, p. 18.

${ }^{7}$ Mbembe, Achille, "Necropolitics", Public Culture, 2003.

${ }^{8}$ Agamben, Giorgio, Estado de excepción, Buenos Aires, AH, 2007.

${ }^{9}$ Anderson, Benedict, Comunidades Imaginadas, reflexiones sobre el origen y la difusión del nacionalismo, México, FCE, 1983.
} 
de un orden político, social y simbólico destinado a definir los contenidos de la nación ${ }^{10}$ significó una reconfiguración de la jerarquía étnico-social anterior en pos de una ficticia homogeneidad nacional. Se desarrollaron así procesos de marcación de mismidad y diferencia, deshistorizantes y esencialistas, continuadores del patrón de poder colonial. Se garantizó, de esta manera, la larga durabilidad de las fronteras raciales, que al interior de una nueva economía política de producción cultural funcionaron como base de las formaciones nacionales de alteridad ${ }^{11}$ resultantes de las articulaciones entre el sistema económico, la estructura social, las instituciones jurídico políticas y los aparatos ideológicos. ${ }^{12}$

La construcción de la nación argentina se basó en lo que Segato llama "terror étnico". Se generó un patrullaje homogeneizador por parte de las instituciones en manos de las elites portuarias e ideológicamente eurocéntricas, con el objetivo de moldear una etnicidad ficticia férreamente uniformizada, vaciada de toda particularidad. ${ }^{13} \mathrm{El}$ pánico a la diversidad expresado en el lema fundacional de Sarmiento "civilización o barbarie" puso en funcionamiento mecanismos institucionales y oficiales, así como estrategias informales de vigilancia, ${ }^{14}$ en pos de una homogeneización ficticia y forzada.

Siguiendo a Funes y Ansaldi, podemos decir que el racismo continuó vigente como cualidad legitimadora pseudocientífica de la matriz de los Estados latinoamericanos (en este caso del Estado argentino) en su proceso de consolidación oligárquica. Proyectó un discurso (acompañado de unas prácticas) que se hace "sentido común" en las sociedad latinoamericanas. ${ }^{15}$ El Estado argentino se entroniza a través del arrinconamiento de las identidades otras consideradas "residuales" o periféricas. ${ }^{16}$ Mantiene para sí, a través de la colonialidad del poder, ${ }^{17}$ la producción de ciudadanos desechables.

La dominación oligárquica vio su fin en la Argentina en el primer cuarto del siglo xx. Sin embargo, las narrativas dominantes, que desde una posición hegemónica "proveen a la nación de una identidad esencializada, establecen sus fronteras externas, su composición interna y proponen el ordenamiento de sus elementos constitutivos, justificando el presente y construyendo un

\footnotetext{
${ }^{10}$ Funes, Patricia y Ansaldi, Waldo, "Patologías y rechazos". El racismo como factor constitutivo de la legitimidad política del orden oligárquico y la cultura política latinoamericana", UDISHAL, Documento de Trabajo, Buenos Aires, cinap, 1995, p. 2.

${ }^{11}$ Briones, Claudia, Cartografias argentinas. Políticas indigenistas y formaciones provinciales de alteridad, Buenos Aires, Geaprona, 2005; Segato, Rita, La nación y sus otros, Buenos Aires, Prometeo Libros, 2007.

${ }^{12}$ Briones, Claudia, Cartografias argentinas. Políticas indigenistas y formaciones provinciales de alteridad, Buenos Aires, Geaprona, 2005.

${ }^{13}$ Segato, Rita, La Nación y sus otros, Buenos Aires, Prometeo Libros, 2007, p. 30.

${ }^{14}$ Idem, p. 51.

${ }^{15}$ Funes, Patricia y Ansaldi, Waldo, op. cit., p. 1.

${ }^{16}$ Segato, Rita, La nación y sus otros, Buenos Aires, Prometeo Libros, 2007, p. 138.

17 Quijano, Aníbal, "Colonialidad del poder, eurocentrismo y América Latina”, En Edgardo Lander (comp.), La colonialidad del saber: eurocentrismo y ciencias sociales. Perspectivas Latinoamericanas, Buenos Aires, Clacso.
} 
pasado legitimador, persistieron en su glorificación de una nación blanca". ${ }^{18}$ En palabras de Briones, ${ }^{19}$ durante el siglo xx el "crisol de razas" argentino devino un caldero con restricciones de ingreso que respondían tanto a consideraciones raciales, como de clase, políticas e ideológicas.

El impulso oficial a la inmigración europea continuó como mecanismo de blanqueamiento a través del cual se "europeizaba a los argentinos y se argentinizaba a los inmigrantes europeos". ${ }^{20}$ Así se popularizó el chiste que dice que "los argentinos descendemos de los barcos", negando el pasado indígena y afrodescendiente de la nación. Éste se basa en un etnocidio que continuó de hecho a través de campañas militares y por derecho a través de campañas oficiales destinadas a invisibilizar las presencias étnicas y raciales.

\section{La escena Del CRIMEN: Buenos Aires, CAPital EUROPEA EN AMÉRICA LATINA}

Para pensar el espacio de la Ciudad Autónoma de Buenos Aires, propongo volver sobre el análisis de Segato, en cuanto define "al territorio como espacio apropiado, delimitado, bajo el control de un sujeto individual o colectivo, marcado por la identidad de su presencia, indisociable de las categorías de dominio y poder, escenario del reconocimiento". ${ }^{21}$

Briones agrega que, a pesar de su arbitrariedad, las fronteras portan su propia materialidad, posibilitando el análisis de las geografias estatales de inclusión y exclusión, según las cuales desde la estatalidad se pondera y ubica en tiempo y espacio la diversidad interior. ${ }^{22} \mathrm{Al}$ interior de Argentina, su capital fue constituida como la ciudad blanca y europea por antonomasia, la posición hegemónica de sus habitantes desde la época colonial en cuanto elite portuaria y el hecho de que los inmigrantes europeos llegaran precisamente a la ciudad porteña. Lo anterior, sumado a la promesa de movilidad ascendente que incluía quedarse allí, convirtió, a través del relato de sus elites, a Buenos Aires en la capital europea en América Latina.

Más acá del relato y de los intereses hegemónicos, los migrantes europeos que llegaban a Buenos Aires no eran exactamente los que la elite sarmientista hubiera querido. La Ley de Residencia sancionada en 1902 da cuenta de la

\footnotetext{
${ }^{18}$ Frigerio, Alejandro, "De la desaparición de los negros a la reaparición de los afrodescendientes: comprendiendo las políticas de las identidades negras, las clasificaciones raciales y de su estudio en Argentina", en Los estudios afroamericanos y africanos en América Latina: herencia, presencia y visiones del otro, Córdoba, Buenos Aires, Clacso, 2008, p. 118.

${ }^{19}$ Briones, Claudia, Cartografias argentinas. Políticas indigenistas y formaciones provinciales de alteridad, Buenos Aires, Geaprona, 2005, p. 27.

${ }^{20}$ Idem, p. 33.

${ }^{21}$ Segato, Rita, La nación y sus otros, Buenos Aires, Prometeo Libros, 2007, pp. 72-73.

${ }^{22}$ Briones, Claudia, Cartografias argentinas. Políticas indigenistas y formaciones provinciales de alteridad, Buenos Aires, Geaprona, 2005, pp. 20-21.
} 
aparición de "elementos indeseables" y de la legalización de su deportación. En 1910 la Ley de Defensa Social va en la misma dirección. En el debate para la aprobación de esta ley, el diputado Ayarragaray propone excluir no sólo a anarquistas y comunistas, sino también a la inmigración amarilla, en sus propias palabras "nosotros necesitamos padres y madres europeas, de raza blanca". ${ }^{23}$

A lo largo del siglo xx, la ciudad de Buenos Aires se convirtió, por su promesa de movilidad ascendente, no en la puerta de entrada, pero sí en el punto de llegada de inmigrantes de los países limítrofes y de conciudadanos de otras latitudes del país, así como de migraciones de otras latitudes planetarias, como Cabo Verde, Medio Oriente y Europa Oriental. Aunque la Ley de Residencia fue derogada en 1958, otros mecanismos y estrategias fueron puestos en funcionamiento en pos de mantener la blanquitud de la ciudad. La falta de oportunidades, la discriminación, la explotación económica y el abandono estatal se desarrollaron para colocar a estos nuevos otros internos en la posición subalterna que, siguiendo los patrones históricos de la ciudad, les "correspondía" ocupar.

Se desarrolló entonces el proceso de "racialización de las relaciones de clase", ${ }^{24}$ que recuerda al racismo estructural definido por Fanon, por el cual se racializó a la subalternidad económica. Con ello, se renovó de manera conservadora "la constitución histórica de esta dinámica de la desigualdad" que se sostiene a través de un proceso de heteroglosia que discrimina sustentado en el cuerpo, en la clase, en la cultura y en la extranjeridad. ${ }^{25} \mathrm{~A}$ través de un proceso de ya larga duración, en Argentina se produjo sobre nuevos colectivos la marca de la otredad, a través de la economía política de producción cultural que resulta de las articulaciones entre el sistema económico, la estructura social, las instituciones jurídico-políticas y los aparatos ideológicos. ${ }^{26}$

Habiendo invisibilizado violentamente a sus otros históricos, descendientes de la esclavitud bajo la premisa de que "en la Argentina no hay negros", se nombró a los migrantes sujetos subalternos bajo el signo de la negritud. A partir de mediados del siglo xx, el término "negro" o "cabecitas negras" se introdujo en el léxico nacional no oficial para hablar de quienes ocupan las posiciones más bajas en la estructura social, "bajo la insistencia de que el término no refiere a lo racial sino a lo meramente socio-económico". ${ }^{27}$ Urresti explica que en estos mecanismos se da la convergencia entre los comportamientos racistas estructurantes de la sociedad colonial que perduran

\footnotetext{
${ }^{23}$ Idem, p. 27.

${ }^{24}$ Margulis, Mario, "La racialización de las relaciones de clase" en Marcelo Margulis y Mario Urresti, $L a$ segregación negada. Cultura y discriminación social, Buenos Aires, Biblios, 1999.

${ }^{25}$ Idem, pp. 38-47

${ }^{26}$ Briones, Claudia, op. cit., pp. 19-20.

${ }^{27}$ Frigerio, Alejandro, op. cit., p. 120.
} 
hasta la época actual y las ideologías y doctrinas racialistas, tempranamente instaladas en América. ${ }^{28}$

La blanquitud de Buenos Aires, amenazada pero mantenida por la fuerza de la ley y la costumbre, perduró entonces desde la Colonia hasta nuestros días. Frigerio explica que "la ceguera cromática de los porteños se corresponde con un proceso doble de invisibilización que se mantiene a nivel microsocial a partir de su construcción constante en las interacciones diarias, como macrosocial en cuanto invisibilización de la presencia del negro en la historia argentina y de sus aportes a la cultura". ${ }^{29}$ En palabras del autor:

La invisibilización de los negros, se produce no solo en la narrativa dominante de la historia argentina, sino en las interacciones sociales de nuestra vida cotidiana. La blanquedad porteña resulta de un proceso socialmente construido y mantenido por una manera de adscribir categorizaciones raciales en las interacciones cotidianas, el ocultamiento de antepasados negros en las familias y el desplazamiento, en el discurso sobre la estratificación y las diferencias sociales, de factores de raza o color hacia los de clase. ${ }^{30}$

La blanquitud y europeidad de Buenos Aires son celosamente custodiadas por sus élites a través de las instituciones oficiales como la escuela, la historiografia y las fuerzas de seguridad, además de las instituciones no oficiales como la discriminación cotidiana y los medios de comunicación. En la Ciudad Autónoma de Buenos Aires, basada en un modelo de segregación espacial, social y racial (en los términos que García Serrano ${ }^{31}$ usa para referirse a Guayaquil), la racialización se produce a diario y la vigilan todos.

\section{EL CASO QUE NOS COMPETE}

La inmigración senegalesa en Argentina forma parte del proceso de migraciones intercontinentales enmarcado en la migración Sur-Sur, y representa el tercer momento en lo que respecta a la migración subsahariana al país, precedido por la trata atlántica colonial y por la inmigración caboverdiana de comienzos del siglo anterior. ${ }^{32}$ Este último momento, caracterizado por

\footnotetext{
${ }^{28}$ Margulis, Mario, "La racialización de las relaciones de clase” en Margulis, Marcelo y Urresti, Mario, $L a$ segregación negada. Cultura y discriminación social, Buenos Aires, Biblios, 1999, p. 49.

${ }^{29}$ Frigerio, Alejandro, op. cit., p. 120.

${ }^{30}$ Frigerio, Alejandro, op. cit., p. 119.

${ }^{31}$ García Serrano, Fernando, "Geografía de la exclusión y negación ciudadana: el pueblo afrodescendiente de la ciudad de Guayaquil, Ecuador", K. Bidaseca y A. Grimson (coords.), Hegemonía culturaly políticas de la diferencia, Buenos Aires, Clacso, 2013.

${ }^{32}$ Zubrzycki, B., \& Alvarado, L., "Redes y proyectos migratorios de los senegaleses en Argentina", Cadernos CERU, vol. 26, núm. 1. Disponible en: http://www.revistas.usp.br/ceru/article/view/111165
} 
la inmigración proveniente de Senegal pero también, en menor medida, de Malí, Nigeria, Ghana, Sierra Leona, Liberia, Camerún y Guinea, tiene inició en la década de 1990 y se ha acentuado durante la década del 2000. Hoy cuenta con entre 1500 y 10000 personas, el primer número según los medios oficiales; el segundo, según las propias organizaciones representativas de los inmigrantes en cuestión. La mayoría reside en la ciudad de Buenos Aires.

La dificultad de obtener cifras oficiales se debe a que la variable del censo nacional no desagrega la información de los migrantes provenientes de África por países de procedencia y a que la Dirección Nacional de Migraciones carece de un registro claro, ya que la mayoría del colectivo ha ingresado al país por pasos fronterizos no habilitados. A primera vista, esto ya da cuenta de un proceso de invisibilización que se sustenta en una falta de estructura nacional para la migración desde Senegal. Desde el año 2002 y hasta el 2015, "la embajada Argentina en Senegal estuvo cerrada, lo cual (con excepción de quienes tuvieran la posibilidad de viajar a Nigeria para tramitar la ciudadanía argentina), obligaba a ingresar al país ilegalmente desde la Triple Frontera". ${ }^{33}$

En el año 2004, bajo la presidencia de Néstor Kirchner, se promulgaron dos mecanismos jurídicos que deberían haber atenuado las dificultades estructurales de este colectivo migrante: el Plan de regularización migratoria $^{34}$ para quienes residieran en el país al 30 de junio del año en cuestión, y la Ley Migratoria 25.871, ${ }^{35}$ por la cual se establecen las condiciones para solicitar la residencia argentina. El hecho de que la mayoría haya llegado desde el país africano después del 2004 los excluye del primer decreto. En cuanto a la Ley Migratoria, las condiciones para solicitar la residencia permanente resultan excluyentes. Se requieren lazos familiares o conyugales con ciudadanos argentinos.

El caso de la residencia temporaria es marcadamente más inclusivo, pero igualmente en la mayoría de los casos los inmigrantes senegaleses no consiguen entrar en las categorías fijadas, las cuales en su mayoría corresponden a la posesión de un alto capital cultural o económico, o a situaciones límites tales como enfermedades terminales, o la condición de refugiados políticos, con la cual tampoco cumple el colectivo en cuestión. En este sentido, podemos dar cuenta de que su exclusión en el país está sostenida en las instituciones jurídico-políticas, así como lo está en el sistema económico.

\footnotetext{
${ }^{33}$ Kleidermacher, Gisele, "Migración Sur-Sur, senegaleses en la Ciudad de Buenos Aires", Voces del Fénix, diciembre, 2012, p. 113.

${ }^{34}$ La Dirección Nacional de Migraciones de la Nación Argentina desarrolló un programa de regularización especial destinado a los migrantes senegaleses entre enero y julio de 2013.

${ }^{35}$ En el año 2010, y en un marco de integración latinoamericana, se aprobó la ley Ley Migratoria 25.871 por la cual se reestablecieron las condiciones de la política migratoria argentina.
} 
Ambos soportes de su exclusión llevan a los inmigrantes senegaleses a dedicarse casi totalmente al trabajo informal y callejero. La falta de documentación los relega a trabajos mal remunerados que carecen de protección estatal. De esta manera, pasan a ocupar las posiciones más bajas de la estructura social y a formar parte de los grupos estereotipados como ilegales y hasta peligrosos en el aparato ideológico que rige en la ciudad. Asimismo, en coyunturas críticas como la actual, son fuertemente rechazadas por la clase media porteña.

Encontramos entonces la articulación de los cuatro factores que según Briones funcionan como soporte de las formaciones nacionales de alteridad: el sistema económico, la estructura social, las instituciones jurídico-políticas y los aparatos ideológicos. La subalternización comienza en una mala situación económica en su país de origen, que los incita a migrar, y persiste con un aparato jurídico-político que excluye a través del sistema económico. Lo anterior los coloca, otra vez, en la base de la estructura social, condenados a la explotación y a la discriminación que sostienen los aparatos ideológicos dominantes.

En los inmigrantes senegaleses, que amenazan con su negritud a la blanquitud de la ciudad, se articulan las dos lógicas históricas de exclusión locales. $\mathrm{Al}$ ser visiblemente negros, aún sin ser descendientes de los esclavizados en la Colonia, exhiben los rasgos que recuerdan y remiten a la derrota histórica de los pueblos africanos frente a los ejércitos coloniales y su posterior esclavización. ${ }^{36}$ Así excluidos como están, son violentamente incluidos en una dinámica alterofóbica sobre la cual se fundó el Estado-nación.

Convergentemente, su posición socio-económica los somete a la dinámica de racialización de las relaciones de clase por la cual son oscurecidos nuevamente, esta vez no porque les sobre melanina, sino porque les faltan recursos. Visiblemente pobres, jurídicamente ilegales, estereotipadamente peligrosos, ideológicamente subalternos, económicamente explotados y doblemente negros, los inmigrantes senegaleses ocupan en la ciudad porteña el lugar de una presa fácil para el maltrato policial y la indiferencia societal.

\section{LOS SOSPECHOSOS DE SIEMPRE}

En la década de 1990, en un marco global de politización de las identidades étnicas y raciales, en el que convergieron la nueva narrativa de la multiculturalidad y las reivindicaciones étnicas y culturales, los colectivos afrodescendientes de la región se organizaron y movilizaron políticamente, interpelando al Estado en pos de reconocimiento y redistribución. Aunque este proceso no tuvo en Argentina la fuerza que logró en otros países latinoamericanos, y a

\footnotetext{
${ }^{36}$ Segato, Rita, La nación y sus otros, Buenos Aires, Prometeo Libros, 2007, p. 115.
} 
pesar de que la condición de inmigrantes excluía a los colectivos senegaleses de reivindicaciones de tipo étnico, las organizaciones afroargentinas incluyeron a los migrantes en una serie de luchas que lograrían al menos ciertos triunfos simbólicos.

Frente al abandono estatal, la organización social y política surgió como la mejor posibilidad contra la explotación y la invisibilización a la que los inmigrantes africanos, mayoritariamente de Senegal, eran sometidos. Massar Ba llegó a la Argentina en 1995, en el mismo año se fundó, con el objetivo de asistir y orientar a los inmigrantes africanos, la Casa de África en Argentina, de la que años más tarde sería presidente, así como referente de la Asociación de Residentes Senegaleses en Argentina (ARSA) y de la Asociación Daira.

Entre las acciones destinadas a mejorar las condiciones de existencia tanto económicas como sociales y culturales de los colectivos inmigrantes africanos en el país, a las cuales se dedican las organizaciones en cuestión, resaltan las luchas contra tres sospechosos del asesinato e invisibilización del homicidio de Massar Ba: la Policía Metropolitana de la Ciudad de Buenos Aires, los medios hegemónicos de comunicación y el lenguaje que sostiene la discriminación cotidiana.

La Policía Metropolitana de la Ciudad de Buenos Aires se creó en el año 2008 bajo iniciativa de Mauricio Macri, hoy presidente de la nación. Comenzó su actividad en el año 2010. En agosto del 2015, las organizaciones senegalesas en Argentina se movilizaron en una marcha desde el Congreso hasta la Legislatura porteña, denunciando abusos de la Policía Metropolitana. La fuerza en cuestión había realizado repetidos operativos de los que fueron objeto los vendedores ambulantes de la comunidad senegalesa, entre otros. En tales operativos se habían decomisado sus productos, los habían desalojado de sus lugares de trabajo y, según las denuncias, se les había robado dinero y maltratado verbal y físicamente.

En los días anteriores a la marcha, la fuerza metropolitana había realizado allanamientos en domicilios, amenazando con armas de fuego. Anteriormente, la comunidad ya había presentado denuncias en torno a entradas a la fuerza a sus hogares por parte de la Policía Metropolitana, en las que ésta había maniatado a los niños, obligado a las mujeres a desnudarse y a los hombres a firmar papeles que según los mismos no comprendían debido a su desconocimiento del español.

La criminalización y persecución a los vendedores ambulantes se convirtió en un arma del Gobierno de la ciudad y de sus integrantes en su campaña por la presidencia. En palabras del titular de la asociación de comerciantes "El compromiso del PRO (partido presidido por Mauricio Macri) fue que, si ganaban la Presidencia, juntaban la policía para limpiar [...] No es la palabra justa [...] Digamos 'despejar' toda la Ciudad, no sólo la calle Avellaneda. 
Empezaron este verano con el procedimiento en Acoyte y Rivadavia. Siguió hasta la Plaza Flores y ahora nos tocó a nosotros" ${ }^{37}$ El PRo, a través de la Policía Metropolitana, cumplió su promesa, ésta continuó hostigando, amenazando y violentando a la comunidad senegalesa. A la atenta y feroz vigilancia que realiza sobre la ciudad, se le pasó por alto el asesinato del referente senegalés que meses antes marchaba en su contra.

Esa noche en plena ciudad de Buenos Aires, las fuerzas del orden no cumplieron con su palabra de proveer seguridad, prevención y protección a los ciudadanos. ¿Será que en esa promesa no entraban los negros inmigrantes?

El abogado, criminólogo, y escritor latinoamericanista, exministro de la Corte Suprema de Justicia de la República Argentina (durante los gobiernos kichneristas), y actual miembro de la Corte Interamericana de Derechos Humanos, Eugenio Raúl Zaffaroni, sostiene que:

Una gran cuestión para el "futuro de los derechos" es la relación vinculante con la concentración monopólica de la información en los medios nacionales e internacionales. En América Latina, los medios de comunicación se hallan concentrados en grandes monopolios que forman parte del capital financiero transnacional. Usualmente, se afirma que están al servicio del poder económico transnacional, lo que no es cierto: su volumen los lleva directamente a ser parte de éste, en una compleja red de intereses íntimamente compartidos. ${ }^{38}$

En Argentina, los medios de comunicación, constructores de la realidad y el sentido común, están altamente concentrados por el conglomerado perteneciente al Grupo Clarín. En el gobierno anterior a cargo de Cristina Kirchner se sancionó la Ley de Servicios de Comunicación Audiovisual, conocida como Ley de Medios, por la cual se buscaba regular los servicios de comunicación audiovisual a nivel nacional, desconcentrar el sistema y promover la diversidad y pluralidad de contenidos.

"El objetivo de la ley era democratizar y universalizar el acceso, entendiendo a la comunicación como un derecho humano básico y no como un bien económico sujeto a las reglas del mercado". ${ }^{39}$ Según la ley, el Grupo Clarín debería desprenderse de ocho señales de televisión y de 213 licencias a nivel nacional. Sin embargo, evidenciando su posición de poder, el Grupo Clarín impugnó la ley a través de medidas cautelares y no se adecuó a la

\footnotetext{
${ }^{37}$ Freixá, Omar y Máximo, Matias, "Nuestras manos también son blancas. La discriminación en la Argentina", Revista Anfibia, 4 de abril, 2016.

${ }^{38}$ Zaffaroni, Eugenio Raul, "Prólogo", en A. Filippi, Constituciones, dictaduras y democracia. Los derechos y su configuración política, Infojus, Ciudad Autónoma de Buenos Aires, 2015, p. 11.

${ }^{39}$ Ley de Servicios de Comunicación Audiovisual: todo lo que tenés que saber sobre el fallo de la Corte (30/10/2013) en Telam.
} 
misma. Meses después de asumir a la presidencia Mauricio Macri (aliado del Grupo Clarín en su enfrentamiento al gobierno anterior), derogó dicha ley a través de un decreto nacional de urgencia.

El 5 de febrero del 2016, Infojus, el servicio de información jurídico, público y gratuito creado en el año 2012 (en el gobierno de Cristina Kirchner), administrado por la Dirección Nacional del Sistema Argentino de Información Jurídica, dependiente de la Secretaría de Justicia del Ministerio de Justicia y Derechos Humanos, fue desmantelado. Sus archivos, que hasta el día anterior estaban disponibles a través de internet, fueron rápidamente diezmados en casi noventa por ciento.

A pesar de las denuncias y pedidos de explicaciones en torno a la eliminación de alrededor de diez mil documentos sobre temas como violencia institucional, violencia de género, lavado de dinero o civiles imputados en delitos de lesa humanidad, el recientemente electo gobierno de Mauricio Macri ni desmintió el hecho ni revirtió la sorpresiva decisión. La noticia que daba cuenta de la marcha realizada por la comunidad senegalesa denunciando a la Policía Metropolitana contó entre las noticias eliminadas del portal y del registro oficial. Se le invisibilizó.

La madrugada en que mataron a Massar, la ciudad de Buenos Aires amaneció sin ninguna noticia al respecto. Llama la atención debido a la hipercomunicación de la cual sus habitantes - como la mayoría de quienes residen en las grandes urbes del siglo XXI - participan, a través de medios digitales y soportes electrónicos. También sorprende, porque en la Argentina hay una tendencia casi obsesiva a mostrar y repetir reiteradamente en radios, televisiones y diarios las noticias en torno a asesinatos morbosos y crímenes sin resolver.

Sin embargo, el homicidio de Massar no fue ni primera plana ni viñeta pequeña en ningún medio de comunicación. Los porteños, ciegos por elección, desayunamos aquel día ignorantes del crimen que nos manchaba a todos. Hasta que entrado el día las organizaciones representantes de los afrodescendientes y de la comunidad senegalesa marcharon a la fiscalía 7, a cargo de la investigación de la causa. El asesinato a Massar Ba, visibilizado por sus propios compañeros, volvió a ser invisibilizado por los medios de comunicación. No figuró entre las noticias del día, y en los días y semanas posteriores fue relevada por medios independientes que debido a la derogación de la Ley de Medios, tienen poco alcance y escasa difusión. En el homicidio a Massar se articularon macabramente la dos lógicas de la invisibilización: la acostumbrada ceguera cromática porteña y la invisibilizante narrativa dominante en manos de los medios de comunicación hegemónicos.

Voloshinov $^{40}$ explica que las significaciones de una sociedad están configuradas siempre por discursos socioculturales que luchan por la definición

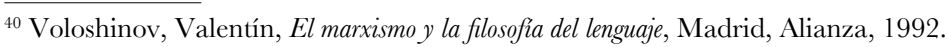


en cada signo. Este último es unidad de identidad y diferencia. Así, subyace a cada signo la lucha por su acentuación y valoración, en la que los grupos enfrentados intentan hegemonizar su sentido. De esta forma, el lenguaje se convierte en un arma valiosa de los grupos dominantes, al hegemonizar el sentido de los signos imponiendo un sentido racista y conservador. Los procesos de subalternización se reproducen a través del habla, produciendo sentidos que se repiten e incorporan inconscientemente. Tal como explica Grimson, en la lengua se establecen las desigualdades categoriales en cada sociedad, a través de la construcción de tipologías para clasificar a la población. ${ }^{41}$ En sociedades hipercomunicadas, los medios de comunicación se convierten en grandes productores de sentido, reproductores de desigualdad que se consolidan en cada palabra.

En octubre del año 2015, todavía bajo el mandato de Cristina Kirchner, la Defensoría del Público de Servicios de Comunicación Audiovisual organizó una Mesa de trabajo en la que participaron las organizaciones representantes de los afrodescendientes y de los inmigrantes de África (Massar Ba incluido), así como representantes de instituciones oficiales y de espacios académicos. Llevó el título “¿Qué decimos cuando decimos 'negro' en los servicios de comunicación audiovisual? Hacia un debate sobre racismo, identidades y pluralismo". El objetivo del encuentro fue analizar los usos del lenguaje, los imaginarios, la estereotipación y las categorías estigmatizantes en los medios audiovisuales, así como la discriminación mediática por invisibilización. Se analizó el concepto de negritud y afrodescendencia y se resaltó la importancia de la diversidad de voces en los medios de comunicación.

Conscientes todos los presentes de la importancia de la lucha por el signo, se formuló el "decálogo de buenas prácticas en relación al tratamiento mediático audiovisual del colectivo de afrodescendientes en Argentina". Este promovía la visibilización de la presencia y los aportes de afrodescendientes y africanos a la nación Argentina, histórica y actualmente, así como del día 8 de noviembre, "Día Nacional de las/os Afroargentinas/os y de la Cultura afro", la difusión de los aspectos positivos de la comunidad, la inclusión de los afrodescendientes y africanos en roles de conducción en los medios de comunicación, así como de sus organizaciones en cuanto fuente de consulta y opinión, y de la diversidad étnica y racial en las publicidades.

Análogamente, se buscaba evitar la vinculación de la palabra negro con prácticas ilegales y clandestinas o con ideas y descripciones consideradas como socialmente negativas, tales como "trabajo en negro" para referirse al trabajo ilegal, "negrear" para dar cuenta de la acción de explotar, "día negro", "suerte negra", los cuales agregan a la palabra negro una carga negativa.

\footnotetext{
${ }^{41}$ Grimson, Alejandro, "La muerte con sangre entra", en Karina Bidaseca y Alejandro Grimson (coord.), Hegemonía cultural y políticas de la diferencia, Buenos Aires, Clacso, 2013, p. 77.
} 
Los asistentes dieron cuenta de la discriminación y de la invisibilización de la que eran objeto, oficial, mediática y cotidianamente, y del rol fundamental que ocupaba el lenguaje en tal proceso de subalternización. Abogaron por la palabra "afrodescendiente" para ser nombrados y por una inclusión tanto discursiva como real. A pesar de las opiniones compartidas, la derogación de la Ley de Medios dio por tierra mucho de lo trabajado aquel día. Aunque el término "afrodescendiente" se impuso en los centros académicos, en el día a día porteño la categoría "negro" se sigue escuchando y reproduciendo. Sin embargo, el día que mataron a Massar, ni negro ni afrodescendiente, el lenguaje, como arma privilegiada de los medios de comunicación, fue utilizado en su forma más reaccionaria. Se hizo uso del más rotundo y completo silencio.

\section{El VEREDICTO}

Zaffaroni sostiene que en la actualidad atravesamos en América Latina un proceso de "genocidio por goteo", en el que el rol de los medios oligopolizados de comunicación es tanto el de ocultar la violencia como el de inventarla e incentivarla, con el objetivo de "montar un poder represivo, mortífero y brutal". ${ }^{42}$ Este mismo poder es el que Mbembe califica como necropolítico, el cual funciona a través de la producción de vidas desechables, nudas vidas (en términos de Agamben), disponibles para hacer morir. Aunque este poder no está legalizado, funciona en los márgenes de la legalidad. No es perseguido ni castigado, marca de antemano a individuos cuya carencia de ciudadanía o ciudadanía de segunda categoría posibilita su disponibilidad para un crimen sin castigo. Tal como mostramos en este artículo, el régimen necropolítico tiene larga data en nuestro país. Produce colectivos subalternos carentes de derechos o dispuestos para desaparecer tanto física como simbólicamente. El asesinato a Massar Ba da cuenta de la persistencia y actualidad de tal régimen, al interior del cual el referente senegalés fue asesinado físicamente, y vuelto a matar simbólicamente a través de la invisibilización del crimen.

En el artículo "La muerte con sangre entra", Grimson analiza, entre otras cosas, la relación entre los asesinatos políticos y las crisis institucionales en la Argentina a partir del 2000. Resalta el hecho de que en el caso del asesinato de Roberto Lopéz, un manifestante Qom en Formosa, a manos de la policía, la muerte no generó tal crisis, y lo relaciona con el carácter periférico tanto de la provincia en cuestión, como con el carácter subalterno del asesinado. Declara que su muerte fue más tipológica que política.

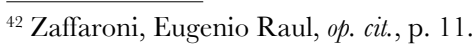


Me permito tomar su artículo para analizar también el caso de Massar, donde el carácter central de Buenos Aires daría a pensar en una mayor repercusión en los medios de comunicación y en las organizaciones políticas. El hecho de que Massar fuera un dirigente comunitario con cargos políticos involucrado en luchas sociales contra actores e instituciones dominantes nos permite clasificarlo como un asesinato político. Pero el carácter subalterno de su posición social, producto del proceso histórico e imperante de racialización, nos incita a calificar, también, su asesinato y su total invisibilización, mayor aún que la del caso analizado por Grimson, para el cual hubo acusados e imputados, como un asesinato tipológico.

$\mathrm{El}$ asesinato de Massar Ba, aparece como el crimen perfecto. El homicida no necesitó esconderse; la víctima era invisible hace siglos. El tan repetido lema de la Declaración Miranda, por el cual el imputado tiene derecho a guardar silencio, encuentra en este caso su máximo paroxismo. Es el silencio de la sociedad toda, histórico, colectivo y estructural, el que nos declara culpables.

\section{REFERENCIAS}

Agamben, Giorgio, Estado de excepción, Buenos Aires, AH, 2007.

Agamben, Giorgio, Homo sacer. El poder soberano y la nuda vida, Valencia, Pre-textos, 2003. Anderson, Benedict, Comunidades Imaginadas, reflexiones sobre el origen y la difusión del nacionalismo, México, FCE, 1983.

Funes, Patricia y Ansaldi, Waldo, "Patologías y rechazos". El racismo como factor constitutivo de la legitimidad política del orden oligárquico y la cultura política latinoamericana, UDISHAL, Documento de Trabajo / 1, Serie III, Buenos Aires, CINAP, 1995.

Braudel, Fernand, "La larga duración", en Fernand Braudel, La Historia y las Ciencias Sociales, Madrid, Alianza, 1970.

Briones, Claudia, Cartografias argentinas. Politicas indigenistas y formaciones provinciales de alteridad, Buenos Aires, Geaprona, 2005.

De Oto, Alejandro, Quintana, María Marta, "Biopolítica y colonialidad. Una lectura crítica de homo sacer", Tabula Rasa, enero-junio, 2010.

Fanon, Frantz, Los condenados de la tierra, Buenos Aires, FCE, 1983.

Foucault, Michel, Defender la sociedad, Buenos Aires, FCE, 2000.

Freixá, Omar y Máximo, Matías, "Nuestras manos también son blancas. La discriminación en la Argentina", Revista Anfibia, 2016, 4 de abril.

Frigerio, Alejandro, "De la desaparición de los negros a la reaparición de los afrodescendientes: comprendiendo las políticas de las identidades negras, las clasificaciones raciales y de su estudio en Argentina", en Los estudios afroamericanos y africanos en América Latina: herencia, presencia y visiones del otro, Córdoba, Buenos Aires, Clacso, 2008.

García Serrano, Fernando, "Geografía de la exclusión y negación ciudadana: el pueblo afrodescendiente de la ciudad de Guayaquil, Ecuador", K. Bidaseca y A. Grimson (coord.), Hegemonía cultural y políticas de la diferencia, Buenos Aires, Clacso, 2013. 
Grimson, Alejandro, "La muerte con sangre entra", en Karina Bidaseca, y Alejandro Grimson (coords.), Hegemonía cultural y políticas de la diferencia, Buenos Aires, Clacso, 2013.

Grüner, Eduardo, La oscuridad y las luces. Capitalismo, cultura y revolución, Buenos Aires, Edhasa, 2010.

Kleidermacher, Gisele, "Migración Sur-Sur, senegaleses en la Ciudad de Buenos Aires", Voces del Fénix, diciembre, 2012.

Margulis, Mario, "La racialización de las relaciones de clase" en Marcelo Margulis y Mario Urresti, et al, La segregación negada. Cultura y discriminación social, Buenos Aires, Biblos, 1999.

Mbembe, Achille, "Necropolitics", Public Culture, vol. 15, núm. 1.

Quijano, Aníbal, "Colonialidad del poder, eurocentrismo y América Latina", en Edgardo Lander, La colonialidad del saber: eurocentrismo y ciencias sociales. Perspectivas latinoamericanas, Buenos Aires, Clacso.

Segato, Rita, La nación y sus otros, Buenos Aires, Prometeo Libros, 2007.

Voloshinov, Valentín, El marxismo y la filosofia del lenguaje, Madrid, Alianza, 1992.

Zaffaroni, Eugenio Raúl, "Prólogo", en A. Filippi, Constituciones, dictaduras y democracia. Los derechos y su configuración política, Buenos Aires, Infojus, 2015.

Zubrzycki, B., \& Alvarado, L., "Redes y proyectos migratorios de los senegaleses en Argentina", Cuadernos CERU, vol. 26, núm. 1. Disponible en: http://www. revistas.usp.br/ceru/article/view/111165 